\title{
BCS-BEC Crossover and Chiral Anomaly in $p$-Wave Superfluids with the Symmetry of A1-Phase
}

\author{
M.Y. Kagan • D.V. Efremov
}

Received: 16 January 2009 / Accepted: 22 October 2009 / Published online: 5 December 2009

(C) The Author(s) 2009. This article is published with open access at Springerlink.com

\begin{abstract}
We solve the Leggett equations for BCS-BEC crossover in the 3D resonance $p$-wave superfluid with the symmetry of A1-phase. We calculate sound velocity, normal density and specific heat for the BCS-domain $(\mu>0)$, BEC-domain $(\mu<0)$ as well as close to important point $\mu=0$ in $100 \%$ polarized case. We find the indications of quantum phase-transition close to the point $\mu(T=0)=0$. Deep in BCS and BEC-domains the crossover ideas of Leggett and Nozieres, Schmitt-Rink work pretty well.

We discuss the spectrum of orbital waves, the paradox of intrinsic angular momentum and complicated problem of chiral anomaly in BCS A1-phase at $T=0$. We present two different approaches to a chiral anomaly: one based on supersymmetric hydrodynamics, another one on the formal analogy with Dirac equation in quantum electrodynamics (QED theory). We evaluate the damping of nodal fermions due to different decay processes in superclean case at $T=0$ and find that we are in a ballistic regime $\omega \tau \gg 1$. We propose to use aerogel or nonmagnetic impurities to reach hydrodynamic regime $\omega \tau \ll 1$ at $T=0$.

We discuss the concept of spectral flow and exact cancellations between timederivatives of anomalous and quasiparticle currents in the equation for the conservation of total linear momentum. We propose to derive and solve a kinetic equation for nodal quasiparticles both in the hydrodynamic and in the ballistic regimes to demonstrate this cancellation explicitly. We briefly discuss the role of the other residual
\end{abstract}

M.Y. Kagan (凶)

P.L. Kapitza Institute for Physical Problems, Kosygina st. 2, 119334 Moscow, Russia

e-mail: kagan@kapitza.ras.ru

D.V. Efremov

Max Planck Institute for Solid State Research, Heisenbergstrasse 1, 70569 Stuttgart, Germany

D.V. Efremov

Max-Planck-Institut für Physik Komplexer Systeme, Nöthnitzer Str. 38, 01187 Dresden, Germany 
interactions different from damping and invite experimentalists to measure the spectrum and damping of orbital waves in A-phase of ${ }^{3} \mathrm{He}$ at low temperatures.

Keywords BCS-BEC crossover $\cdot$ Feshbach resonance $\cdot$ Superfluid ${ }^{3} \mathrm{He}-\mathrm{A} \cdot$ Chiral anomaly $\cdot$ Spectral flow

PACS 67.30.H- $\cdot$ 67.85.Lm $\cdot$ 74.20.Rp

\section{Introduction}

The first experimental results on $p$-wave Feshbach resonance [1-6] in ultracold fermionic gasses ${ }^{40} \mathrm{~K}$ and ${ }^{6} \mathrm{Li}$ make the field of quantum gasses closer to the interesting physics of superfluid ${ }^{3} \mathrm{He}$ and the physics of unconventional superconductors such as $\mathrm{Sr}_{2} \mathrm{RuO}_{4}$. In this context it is important to build the bridge between the physics of ultracold gasses and the low-temperature physics of quantum liquids and anomalous superconductors and thus to enrich both communities with the experience and knowledge accumulated in each of these fields. The purpose of the present paper is first of all to describe the transition from the weakly bound cooper pairs with $p$-wave symmetry to strongly bound local $p$-wave pairs (molecules) and try to reveal the nontrivial topological effects connected with the presence of the nodes in the superfluid gap of the $100 \%$-polarized $p$-wave A1-phase in 3D. Note, that the A1phase symmetry is relevant both to ultracold Fermi-gasses in the regime of $p$-wave Feshbach resonance and to superfluid ${ }^{3} \mathrm{He}-\mathrm{A}$ in the presence of large magnetic field or large spin-polarization. We pay the special attention to the spectrum of collective excitations and to the superfluid hydrodynamics of the A1-phase at $T=0$, where the topological effects are very pronounced, especially in the BCS-domain. We propose the experimental verification of the different approaches connected with the complicated problem of chiral anomaly and mass-current nonconservation in superfluid A1-phase of ${ }^{3} \mathrm{He}$ in superclean case and in the presence of aerogel as well as for the A1 $p$-wave condensates in magnetic traps in the presence of Josephson tunneling currents.

Our paper is organized as follows. Section 1 provides an Introduction. In Sect. 2 we briefly comment on the recent experiments on $p$-wave Feshbach resonance and describe the global phase-diagram for $100 \%$-polarized $p$-wave resonance superfluids in 3D. In Sect. 3 we describe the quasiparticle spectrum and nodal points in A1-phase. In Sect. 4 we solve mean-field Leggett equations for triplet superfluids with the symmetry of A1-phase at $T=0$ and study the behavior of superfluid gap $\Delta$, chemical potential $\mu$, and sound velocity $c_{S}$ deep in BCS- $(\mu>0)$ and BEC-domains $(\mu<0)$ as well as close to the interesting point $\mu=0$. In Sect. 5 we study the temperature behavior of the normal density $\rho_{n}$ and specific heat $C_{v}$ in BCS-domain, in BEC-domain, and close to $\mu=0$, where we find the indications of quantum phase-transition.

In Sect. 6 we describe the orbital waves spectrum in BCS- and BEC-domains of the A1-phase and describe the complicated problem of chiral anomaly (mass-current nonconservation) which exists in the superfluid hydrodynamics of A1-phase in BCSdomain at $T \rightarrow 0$. In Sects. 7 and 8 we present two different approaches to the calculation of anomalous current: first one based on supersymmetric hydrodynamics [7] 
and the second one - on the analogy with Dirac equation in quantum electrodynamics (QED) [8-12]. Note that both approaches are very general. The first one is based on the inclusion of fermionic Goldstone mode in low-frequency hydrodynamic action [7]. It can be useful for all nodal superfluids and superconductors with zeroes of the superconductive gap such as ${ }^{3} \mathrm{He}-\mathrm{A}, \mathrm{Sr}_{2} \mathrm{RuO}_{4}, \mathrm{UPt}_{3}, \mathrm{UNi}_{2} \mathrm{Al}_{3}, \mathrm{U}_{1-x} \mathrm{Th}_{x} \mathrm{Be}_{13}$ and so on [13]. The second approach is also very nice and general. It is connected with the appearance of the Dirac-like spectrum of fermions with zero mode [8-12] which also arises in many condensed-matter systems such as ${ }^{3} \mathrm{He}-\mathrm{A}$, chiral superconductor $\mathrm{Sr}_{2} \mathrm{RuO}_{4}$, organic conductor $\alpha$-(BEDT-TTF) ${ }_{2} \mathrm{I}_{3}$, 2D semiconductors, or recently discovered graphene [13-17]. In Sect. 9 we evaluate the damping in superclean A1-phase at $T=0$ due to different decay processed and come to the conclusion that we are in a ballistic regime $\omega \tau \gg 1$ at $T=0$. We propose to use aerogel or nonmagnetic impurities to reach hydrodynamic regime $\omega \tau \ll 1$. We discuss the concept of spectral flow and exact cancellations of anomalies between time-derivatives of anomalous and quasiparticle currents in the equation of the conservation of total linear momentum. We also propose to derive both in a hydrodynamic and in a ballistic regimes a kinetic equation for nodal quasiparticles and to demonstrate this cancellation explicitly. In Sect. 10 we provide our conclusions and acknowledgements. We also invite experimentalists to measure the spectrum and damping of orbital waves in ${ }^{3} \mathrm{He}$-A phase at low temperatures $T \ll T_{C}$ and thus to help to resolve the paradox of the orbital momentum. We also propose to extend the measurements of orbital inertia and orbital viscosity in non-singular vortex textures in A-phase $[18,19]$ to low temperatures with the help of the creation of spin-polarization. Finally we propose to measure a Josephson current between two 2D films of axial and planar phases with an attempt to extract directly the difference between topological charges $\Delta Q=1$ in these phases.

\section{Feshbach Resonance and Phase-Diagram for 100\%-Polarized $p$-Wave Resonance Superfluids}

In the first experiments on $p$-wave Feshbach resonance the experimentalists measure the molecule formation in the ultracold fermionic gas of ${ }^{6} \mathrm{Li}$-atoms close to resonance magnetic field $B_{0}$ [1-5].

In the last years the analogous experiments on $p$-wave molecules formation in spin-polarized fermionic gas of ${ }^{40} \mathrm{~K}$-atoms were started [6]. The lifetime of $p$-wave molecules is rather short yet [1-6]. However the physicists working in ultracold gasses have started intensively to study the huge bulk of experimental and theoretical wisdom accumulated in the physics of superfluid ${ }^{3} \mathrm{He}$ and anomalous complex superconductors (see [20, 21]).

To understand the essence of $p$-wave Feshbach resonance we should recollect the basic formula on $p$-wave scattering amplitude in vacuum from [22-25]:

$$
f_{l=1}(E)=\frac{p p^{\prime}}{\frac{1}{V_{p}}+\frac{2 m E}{\pi r_{0}}+i(2 m E)^{3 / 2}},
$$


Fig. 1 (Color online) The sketch of the $p$-wave Feshbach resonance. For $B=B_{0}$ the scattering volume $V_{p}$ diverges

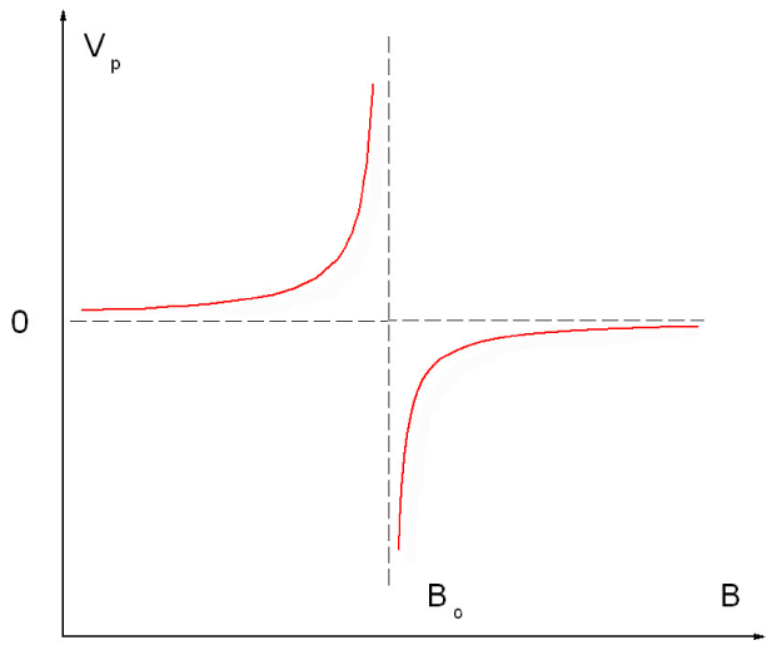

where $l=1$ is an orbital momentum in the $p$-wave channel, $E$ is a two particle energy, $V_{p}=r_{0}^{2} a_{p}$ is scattering volume, $a_{p}$ is $p$-wave scattering length, $r_{0}$ is the range of the interaction, $p, p^{\prime}$ are incoming and outcoming momenta. For Feshbach resonance in fermionic systems $p \sim p^{\prime} \sim p_{F}$ and usually $p_{F} r_{0}<1$. The scattering length $a_{p}$ and hence the scattering volume $V_{p}$ is divergent in the resonance magnetic field $B_{0}$ (see Fig. 1) $1 / V_{p}=1 / a_{p}=0$. The imaginary part of the scattering amplitude $f_{p}$ is small and nonzero only for positive energies $E>0$, so $p$-wave Feshbach resonance is intrinsically narrow. Note that for negative energies $E<0$ there is a molecular bound state:

$$
\left|E_{b}\right|=\frac{\pi r_{0}}{2 m V_{p}}=\frac{\pi}{2 m r_{0} a_{p}} .
$$

In the unitary limit the molecular binding energy $\left|E_{b}\right| \rightarrow 0$.

The first theoretical articles on $p$-wave Feshbach resonance often deal with meanfield two-channel description of the resonance [23-25]. In our paper we will study $p$-wave Feshbach resonance in the framework of one-channel description, which is more close to the physics of superfluid ${ }^{3} \mathrm{He}$ and captures rather well the essential physics of BCS-BEC crossover in $p$-wave superfluids.

In magnetic traps (in the absence of the so-called dipolar splitting) people usually study fully $(100 \%)$ polarized gas or more precisely-one hyperfine component of the gas. On the language of ${ }^{3} \mathrm{He}$ they study the pairs with $S_{t o t}=S_{z}^{t o t}=1$, or $|\uparrow \uparrow\rangle$-pairs. In our paper we will consider $p$-wave triplet A1-phase in 3D where just $S_{\text {tot }}=S_{z}^{\text {tot }}=1$.

The qualitative picture of the global phase-diagram of the BCS-BEC crossover in $100 \%$-polarized A1-phase is presented in Fig. 2. In gross features it resembles the phase-diagram of the BCS-BEC crossover for $s$-wave pairing (see [26-29] for more details). However there is a very interesting question about the origin of the point $\mu(T=0)=0$ for $3 \mathrm{D}$ A1-phase. We will show that in the point $\mu(T=0)=0$ we probably deal with a quantum phase-transition [30, 31]. 
Fig. 2 Qualitative picture of the BCS-BEC crossover in $100 \%$-polarized A1-phase in the axis $T$ (temperature) and $1 / \lambda_{p}$-inverse gas parameter for $p$-wave superfluids $\left(\lambda_{p}=V_{p} p_{F}^{3}, V_{p}\right.$ is scattering volume), $\mu$ is chemical potential, $a_{p}$ is scattering length $\left(V_{p}=r_{0}^{2} a_{p}\right)$. We indicate the line where $\mu(T)=0$ and a quantum phase-transition point $\mu(T=0)=0$

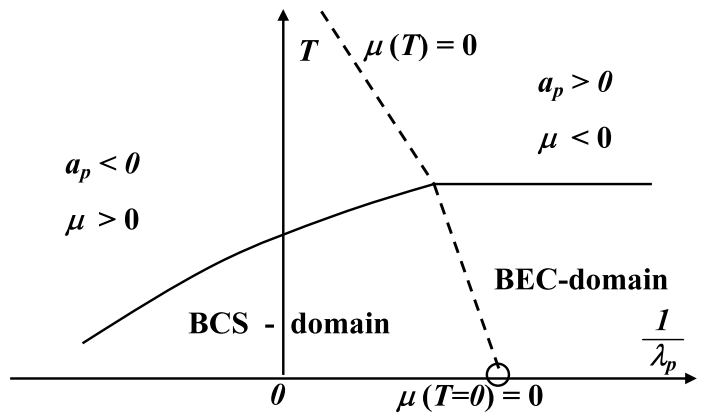

BCS-domain where chemical potential $\mu>0$, occupies on the global phasediagram the region of negative values of the gas parameter $\lambda_{p}=V_{p} p_{F}^{3}<0$ (or the negative values of the scattering length $a_{p}$ ). It stretches also to the small positive values of the inverse gas parameter $1 / \lambda_{p} \leq 1$ and is separated from the BEC-domain (where $\mu<0$ and the inverse gas parameter is large and positive $1 / \lambda_{p} \geq 1$ ) by the line $\mu(T)=0$. Usually in the regime of Feshbach resonance the density of "up" spins $n=p_{F}^{3} / 6 \pi^{2}$ is fixed. Deep inside BCS-domain (for small absolute values of the gas parameter $\left|\lambda_{p}\right| \ll 1$ ) we have the standard BCS-like formula for the critical temperature of the A1-phase:

$$
T_{C p}=0.1 \varepsilon_{F} e^{-\frac{\pi}{2|\lambda p|}},
$$

where the preexponential factor for the $100 \%$ polarized A1-phase is defined by second order diagrams of Gor'kov and Melik-Barchudarov type [32] and approximately equals to $0.1 \varepsilon_{F}[33-36]$.

Deep in BEC-domain $\left(\lambda_{p} \ll 1\right)$ the well-known formula of Einstein is working in principal approximation for Bose-condensation of $p$-wave molecules with the density $n / 2$ and the mass $2 m$ :

$$
T_{C p}=3.31 \frac{(n / 2)^{2 / 3}}{2 m} .
$$

In the unitary limit $1 / \lambda_{p}=0$. Hence here $T_{C p} \approx 0.1 \varepsilon_{F}$ and we are still in BCSregime (see [26-29]). In the rest of the paper we will consider low temperatures $T \ll T_{C}$, so we will work deep in the superfluid parts of BCS and BEC-domains of the A1-phase.

\section{Quasiparticle Energy and Nodal Points in A1-Phase}

For standard $s$-wave pairing the quasiparticle spectrum reads:

$$
E_{p}=\sqrt{\left(\frac{p^{2}}{2 m}-\mu\right)^{2}+\Delta_{0}^{2}}
$$


Fig. 3 The topology of the superfluid gap in A1-phase. $\theta$ is the angle between momentum $\vec{p}$ and the axis of orbital momentum quantization $\vec{l}=\vec{e}_{z}$. There are two nodes in the quasiparticle spectrum corresponding to the south and north poles

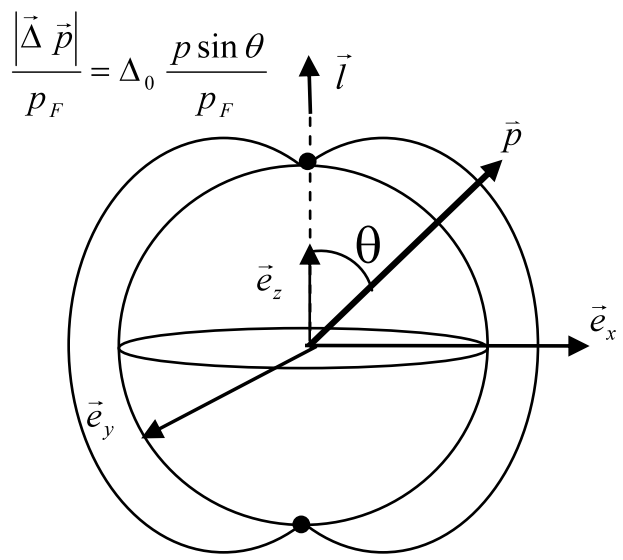

It has no zeroes (no nodes), so the topology of the $s$-wave pairing problem is trivial. For triplet A1-phase however:

$$
E_{p}=\sqrt{\left(\frac{p^{2}}{2 m}-\mu\right)^{2}+\frac{|\vec{\Delta} \vec{p}|^{2}}{p_{F}^{2}}},
$$

where $\vec{\Delta}=\Delta_{0}\left(\vec{e}_{x}+i \vec{e}_{y}\right)$-is the complex order parameter in A1-phase, $\Delta_{0}$ is the magnitude of the superfluid gap. In fact: $|\vec{\Delta} \vec{p}|^{2}=\Delta_{0}^{2} p^{2} \sin ^{2} \theta=\Delta_{0}^{2}[\vec{p} \vec{l}]^{2}$, where $\vec{l}=$ $\left[\vec{e}_{x}, \vec{e}_{y}\right]$-is the unit vector of orbital momentum (see Fig. 3). Note that $p_{F}$ is fixed by fixed density $n$. Angle $\theta$ is the angle between momentum $\vec{p}$ and the orbital momentum quantization axis $\vec{l}=\vec{e}_{z}$.

For $\mu>0$ (BCS-domain) there are two nodes in the spectrum for $p^{2} / 2 m=\mu$ and for $\theta=0$ or $\pi$. For $\mu<0$ (BEC-domain) there are no nodes. The important point $\mu=0$ is a boundary between the totally gapped BEC-domain and the BCS-domain with two nodes of the quasiparticle spectrum corresponding to the south and north poles of Fig. 3. Often this point for $T=0$ is called the point of topological quantum phase-transition [37-41].

\section{Leggett Equations for A1-Phase}

The Leggett equations for the $100 \%$-polarized A1-phase in 3D are the evident generalization of the standard Leggett equations for the $s$-wave BCS-BEC crossover [26-29, 42]. The first equation reads:

$$
n=\frac{p_{F}^{3}}{6 \pi^{2}}=\int_{0}^{1 / r_{0}} \frac{p^{2} d p}{2 \pi^{2}} \int_{-1}^{1} \frac{d x}{2}\left(1-\frac{\xi_{p}}{E_{p}}\right) \frac{1}{2},
$$

where $\xi_{p}=\left(\frac{p^{2}}{2 m}-\mu\right), E_{p}=\sqrt{\xi_{p}^{2}+\frac{\Delta_{0}^{2} p^{2}}{p_{F}^{2}} \sin ^{2} \theta}$-is a quasiparticle spectrum, $x=$ $\cos \theta$. This equation defines the chemical potential $\mu$ for fixed density $n$. 
The second self-consistency equation defines the magnitude of the superfluid gap $\Delta_{0}$. It reads:

$$
-\pi m \operatorname{Re} \frac{1}{f_{l=1}(2 \mu)}=\int_{-1}^{1} \frac{d x}{2} \int_{0}^{1 / r_{0}} p^{4} d p\left\{\frac{1}{E_{p}}-\frac{1}{\xi_{p}}\right\},
$$

where $\operatorname{Re} \frac{1}{f_{l=1}(2 \mu)}=\left(\frac{1}{V_{p}}+\frac{4 m \mu}{\pi r_{0}}\right)$-is a real part of an inverse scattering amplitude in $p$-wave channel for total energy $E=2 \mu$ of colliding particles. This energy is relevant for pairing problem, so $f_{l=1}(E)$ should be replaced by $f_{l=1}(2 \mu)$ in Legget equations. In (7) $V_{p}=r_{0}^{2} a_{p}$.

The solution of Leggett equations yields deep in the BCS-domain:

$$
\Delta_{0} \sim \varepsilon_{F} e^{-\frac{\pi}{2|\lambda p|}} \sim T_{C p} ; \quad \mu \approx \varepsilon_{F}>0 .
$$

The sound velocity reads in $3 \mathrm{D}$ :

$$
c_{S}=\left(\frac{n}{m} \frac{d \mu}{d n}\right)^{1 / 2}=\frac{v_{F}}{\sqrt{3}} .
$$

For $1 /\left|\lambda_{p}\right|=0: \Delta_{0} \sim \varepsilon_{F}$ and hence unitary limit is still inside BCS-domain.

Deep in BEC-domain:

$$
\Delta_{0} \approx 2 \varepsilon_{F} \sqrt{p_{F} r_{0}} \ll \varepsilon_{F} \quad \text { for } p_{F} r_{0} \ll 1,
$$

and chemical potential $\mu=-\frac{\left|E_{b}\right|}{2}+\frac{\mu_{B}}{2}<0$, where as we already mentioned:

$$
\left|E_{b}\right|=\frac{\pi}{2 m r_{0} a_{p}}
$$

is a binding energy of a triplet pair (molecule).

Accordingly:

$$
\mu_{B} \approx \frac{4 \varepsilon_{F}}{3} \sqrt{p_{F} r_{0}}
$$

is a bosonic chemical potential which governs the repulsive interaction between two $p$-wave molecules [26-29].

The sound velocity deep in BEC-domain reads:

$$
c_{S}=\left(\frac{n_{B}}{2 m} \frac{d \mu_{B}}{d n_{B}}\right)^{1 / 2} \approx \frac{v_{F}}{\sqrt{3}} \sqrt{p_{F} r_{0}} \ll v_{F} \quad \text { for } p_{F} r_{0} \ll 1,
$$

where $n_{B}=n / 2$ is bosonic density.

Close to the interesting point $\mu \rightarrow 0$ (more rigorously $|\mu|<\Delta_{0}^{2} / \varepsilon_{F}$ ) we have:

$$
\Delta_{0}(\mu=0)=2 \varepsilon_{F} \sqrt{p_{F} r_{0}}
$$

for the magnitude of the superfluid gap. 
For the gas parameter $\lambda_{p}$ in the point $\mu=0$ we have:

$$
\lambda_{p}(\mu=0)=\frac{3 \pi}{4}>0 .
$$

Hence the interesting point $\mu=0$ is effectively in BEC-domain (in the domain of positive $p$-wave scattering length $a_{p}>0$ ). Accordingly for $\mu=0$ the binding energy:

$$
\left|E_{b}\right|=\frac{4}{3} \varepsilon_{F}\left(p_{F} r_{0}\right) .
$$

The sound velocity squared for $\mu=0$ reads:

$$
c_{S}^{2}=\frac{v_{F}^{2}}{3}\left(p_{F} r_{0}\right)
$$

and coincides with the expression (10) obtained deep in BEC-domain. The careful analysis of Leggett equations close to the point $\mu=0$ shows that the derivative $\frac{\partial \Delta}{\partial \mu}$ also have no singularities at this point. The second derivative $\frac{\partial^{2} n}{\partial \mu^{2}}$ is also continuous at $\mu=0$, so the anomaly will appear only in higher derivatives in qualitative agreement with numerical calculations of $[43,44]$ in 3D.

At the same time the careful analysis of compressibility in 2D case [45-47] shows continuous behavior but with a kink already in $\frac{\partial n}{\partial \mu}$ in $100 \%$-polarized $\left(p_{x}+i p_{y}\right)$ phase for $\mu=0$ on the level of analytical calculation as well as in numerics [43, 44].

To be more specific:

$$
\frac{\partial n}{\partial \mu} \propto 1+\frac{\mu \varepsilon_{F}}{\Delta_{0}^{2}}[1-\operatorname{sign} \mu]
$$

and thus $\frac{\partial n}{\partial \mu} \propto 1$ for $\mu \rightarrow+0$, and $\frac{\partial n}{\partial \mu} \propto 1+\frac{2 \mu \varepsilon_{F}}{\Delta_{0}^{2}}$ for $\mu \rightarrow-0$.

\section{Specific Heat and Normal Density at Low Temperatures $T \ll T_{C}$}

In this section we study the thermodynamic functions namely, normal density $\rho_{n}$ and specific heat $C_{v}$ in 3D resonance $p$-wave superfluids with A1-symmetry at low temperatures $T \ll T_{C}$. Our goal is to try to find nontrivial contributions to $\rho_{n}$ and $C_{v}$ from the nodal points on the mean-field level.

\subsection{Specific heat in 3D A1-phase}

The fermionic (quasiparticle) contribution to $C_{v}$ on the mean-field level yields in 3D (see [48]):

$$
C_{v}=\int \frac{\partial n_{0}\left(E_{p} / T\right)}{\partial T} E_{p} \frac{d^{3} \vec{p}}{(2 \pi)^{3}},
$$

where $n_{0}\left(\frac{E_{p}}{T}\right)=\frac{1}{\left(e^{\frac{E_{p}}{T}}+1\right)}$ is quasiparticle distribution function, $E_{p}$ is quasiparticle energy given by (5). 
The calculations yield an expected result:

$$
C_{v} \sim N(0) \frac{T^{3}}{\Delta_{0}^{2}}
$$

deep in the BCS-domain, where $N(0)=\frac{m p_{F}}{2 \pi^{2}}$ is the density of states at the Fermisurface. Deep in the BEC-domain $C_{v}$ is exponential:

$$
C_{v} \sim \frac{(2 m T)^{3 / 2}}{2 \pi^{2}} \frac{E_{b}}{4 T^{2}} e^{-\frac{\left|E_{b}\right|}{2 T}},
$$

where $\left|E_{b}\right|$ is given by (11).

Finally in the interesting region of small $\mu$ and low temperatures $(|\mu| \ll T \ll$ $\Delta_{0}^{2} / \varepsilon_{F}$ and thus for the classical limit $\frac{|\mu|}{T} \rightarrow 0$ ) we have a nontrivial temperature dependence for $C_{v}$ :

$$
C_{v} \sim \frac{(2 m T)^{3 / 2}}{2 \pi^{2}} \frac{\varepsilon_{F} T}{\Delta_{0}^{2}}
$$

Note that in the opposite quantum limit $\frac{T}{|\mu|} \rightarrow 0\left(T \ll|\mu| \ll \frac{\Delta_{0}^{2}}{\varepsilon_{F}}\right)$ we have:

$$
C_{v} \sim \frac{1}{2 \pi^{2}} \frac{\varepsilon_{F} T}{\Delta_{0}^{2}} \frac{T^{2} m^{3 / 2}}{\mu^{1 / 2}}
$$

in BCS-domain and respectively:

$$
C_{v} \sim \frac{(2 m T)^{3 / 2}}{2 \pi^{2}} \frac{|\mu|^{3}}{T^{3}} e^{-\frac{|\mu|}{T}} \frac{\varepsilon_{F} T}{\Delta_{0}^{2}}
$$

in BEC-domain. In this limit $C_{v}$ behaves very differently in BCS and BEC-domains.

For $|\mu| \sim T$ these results (23), (24) coincide by the order of magnitude with (22).

For small $|\mu|$ but intermediate temperatures $|\mu| \ll \Delta_{0}^{2} / \varepsilon_{F} \ll T \ll \Delta_{0}$ we recover a more expected result:

$$
C_{v} \sim \frac{(2 m T)^{3 / 2}}{2 \pi^{2}}
$$

However at these temperatures bosonic contribution (contribution from the sound waves) prevails and yields:

$$
C_{v}^{B} \sim \frac{T^{3}}{c_{S}^{3}} \frac{1}{2 \pi^{2}},
$$

where the sound velocity $c_{s}$ is given by (9) in BCS-domain and by (13), (17) in BECdomain and close to $\mu=0$.

We can see that it is possible to separate a power-law fermionic contribution $C_{v} \sim$ $T^{5 / 2}$ at low temperatures and $C_{v} \sim T^{3 / 2}$ at intermediate temperatures from bosonic one $C_{v}^{B} \sim T^{3}$ close to the important point $\mu=0$. We also see very different behavior of $C_{v}$ in BCS and BEC-domains in the limit $\frac{T}{|\mu|} \rightarrow 0$. 
Analogously in 2D 100\%-polarized $\left(p_{x}+i p_{y}\right)$-phase in quantum limit $T \ll|\mu| \ll$ $\frac{\Delta_{0}^{2}}{\varepsilon_{F}}\left(\frac{T}{|\mu|} \rightarrow 0\right)$ we have for a quasiparticle contribution:

$$
C_{v} \sim \frac{1}{2 \pi} \frac{m \varepsilon_{F}}{\Delta_{0}^{2}} T^{2}
$$

in BCS-domain for $\mu \rightarrow+0$. Note that phonon contribution has the same order of magnitude as a fermionic contribution in BCS-domain.

In BEC-domain for $\mu \rightarrow-0$ :

$$
C_{v} \sim \frac{1}{2 \pi} \frac{m \varepsilon_{F}}{\Delta_{0}^{2}} \frac{|\mu|^{3}}{T} e^{-\frac{|\mu|}{T}} .
$$

Note that both in $3 \mathrm{D}$ and $2 \mathrm{D}$ for $T \neq 0$ we are effectively always in a classical limit $\frac{|\mu|}{T} \rightarrow 0$ since $\mu$ is continuous close to $\mu=0$. Hence the real phase transition takes place only at $T=0$ [37-41].

\subsection{Normal Density in 3D A1-Phase}

The quasiparticle contribution to the normal density in 3D A1-phase yields (see [48]):

$$
\rho_{n}=-\frac{1}{3} \int p^{2} \frac{\partial n_{0}\left(E_{p} / T\right)}{\partial E_{p}} \frac{d^{3} \vec{p}}{(2 \pi)^{3}} .
$$

Deep in the BCS-domain the evaluation of $\rho_{n}$ yields:

$$
\rho_{n} \sim \rho \frac{T^{2}}{\Delta_{0}^{2}}
$$

where $\rho=m n$ is a total mass-density. Note that rigorously speaking equation (30) yields longitudinal component of normal density tensor $\rho_{n l}$. There is also a small transverse contribution $\rho_{n t} \sim T^{4}$ firstly obtained by [37-40].

Deep in the BEC-domain the normal density is exponential and reads:

$$
\rho_{n} \sim \frac{m}{\pi^{2}}(2 m T)^{3 / 2} e^{-\frac{E_{b}}{2 T}}
$$

Finally close to $\mu=0$ at low temperatures $\left(|\mu| \ll T \ll \Delta_{0}^{2} / \varepsilon_{F}\right.$ and thus in the classical limit $\frac{|\mu|}{T} \rightarrow 0$ ) we have:

$$
\rho_{n} \sim \frac{m}{\pi^{2}}(2 m T)^{3 / 2} \frac{\varepsilon_{F} T}{\Delta_{0}^{2}} .
$$

In the opposite quantum limit $\frac{T}{|\mu|} \rightarrow 0\left(T<|\mu|<\frac{\Delta_{0}^{2}}{\varepsilon_{F}}\right)$ we have:

$$
\rho_{n} \sim \frac{m}{\pi^{2}} \frac{\varepsilon_{F} T}{\Delta_{0}^{2}} 2 m T(2 m|\mu|)^{1 / 2}
$$


in BCS-domain and respectively:

$$
\rho_{n} \sim \frac{m}{\pi^{2}} \frac{\varepsilon_{F} T}{\Delta_{0}^{2}} e^{-\frac{|\mu|}{T}} 2 m|\mu|(2 m T)^{1 / 2}
$$

in BEC-domain, so again the behavior of $\rho_{n}$ is very different in BCS and BECdomains in the quantum limit.

For $|\mu| \sim T$ these results (33), (34) coincide by the order of magnitude with (32).

At intermediate temperatures $|\mu| \ll \Delta_{0}^{2} / \varepsilon_{F} \ll T \ll \Delta_{0}$ a normal density yields as expected:

$$
\rho_{n} \sim \frac{m}{\pi^{2}}(2 m T)^{3 / 2} .
$$

However at these temperatures bosonic (phonon) contribution from the sound waves prevails and yields (see [48]):

$$
\rho_{n}^{B} \sim \frac{T^{4}}{c_{s}^{5}},
$$

where $c_{s}$ is again given by (5), (13) and (17) in BCS-, BEC-domain and close to $\mu=0$ respectively. We can again separate a fermionic (quasiparticle) contribution to $\rho_{n}\left(\rho_{n} \sim T^{5 / 2}\right.$ at low temperatures and $\rho_{n} \sim T^{3 / 2}$ at intermediate temperatures) from bosonic one $\left(\rho_{n} \sim T^{4}\right)$ close to the point $\mu=0$. We also see a very different behavior of $\rho_{n}$ in BEC and BCS-domains in the quantum limit $\frac{T}{|\mu|} \rightarrow 0$. The same behavior holds for a $2 \mathrm{D}$ case.

\section{Orbital Waves, Intrinsic Angular Momentum and the Chiral Anomaly in A1-Phase}

The topological effects in A1-phase are really pronounced in the spectrum of orbital waves and in the superfluid hydrodynamics at low temperatures $T \rightarrow 0$ especially in BCS-domain. Here by symmetry requirements we can write the following expression for the total mass-current:

$$
\vec{j}_{\text {tot }}=\vec{j}_{B}+\vec{j}_{\text {an }},
$$

where

$$
\vec{j}_{a n}=-\frac{\hbar}{4 m} C_{0}(\vec{l}, \operatorname{rot} \vec{l}) \vec{l}
$$

is an anomalous current. In BEC-domain $C_{0}=0$ and anomalous current is absent. The reason for that is connected with the fact that the integral $\frac{N(0)}{2} \int d \xi_{p}\left(1-\frac{\xi_{p}}{\left|\xi_{p}\right|}\right)=0$ in BEC-domain (for $\xi_{p}>0$ ), while it is nonzero and defines total density in BCSdomain. However it is a difficult question whether $C_{0}=0$ or not in the BCS-domain.

In the same time $\vec{j}_{B}$ in (37) is a total mass-current in BEC-domain for $p$-wave molecules. It reads:

$$
\vec{j}_{B}=\rho \vec{v}_{S}+\frac{\hbar}{2 m} \operatorname{rot} \frac{\rho \vec{l}}{2},
$$


where $\vec{L}=\frac{\hbar \rho}{2 m} \vec{l}$ is the density of orbital momentum, $\vec{v}_{S}$ is a superfluid velocity.

Anomalous current $\vec{j}_{\text {an }}$ violates conservation law for total mass-current (total linear momentum) $\vec{j}_{\text {tot }}$ since it cannot be expressed as a divergence of some momentum tensor $\prod_{i k}$ :

$$
\frac{\partial j_{t o t}^{i}}{\partial t} \neq-\frac{\partial}{\partial x_{k}}\left(\Pi_{i k}\right) .
$$

Thus the presence of anomalous current destroys the superfluid hydrodynamics of the A1-phase at $T \rightarrow 0$. Its contribution to the equation for total linear momentum (to $\frac{\partial j_{t o t}^{i}}{\partial t}$ ) can be compensated only by adding the term with a relative normal velocity and normal density $\rho_{n}(T=0)\left(\vec{v}_{n}-\vec{v}_{S}\right)$ to the total current $\vec{j}_{\text {tot }}$ already at $T=0$ (see [8-12]). The anomalous current also changes significantly the spectrum of orbital waves. This additional Goldstone branch of collective excitations in the A1-phase is connected with the rotation of $\vec{l}$-vector around perpendicular axis. It is quadratic at low frequencies (A1-phase is called an orbital ferromagnet. It is also spin ferromagnet). However, the coefficient in front of $q^{2}$ is drastically different in BCSand BEC-domains.

In BEC-domain for small $\omega$ and $\vec{q}$ :

$$
\omega \sim \frac{q^{2}}{m}
$$

At the same time in BCS-domain:

$$
\frac{\left(\rho-C_{0}\right)}{\rho} \omega \sim \frac{q^{2}}{m} .
$$

The most straightforward way to get (41) and (42) is to use diagrammatic technique of $[49,50]$ for collective excitations spectrum in $p$-wave and $d$-wave superfluids. If according to [20] the relative difference $\frac{\left(\rho-C_{0}\right)}{\rho} \sim \frac{\Delta_{0}^{2}}{\varepsilon_{F}^{2}} \ll 1$ then the coefficient in front of $\omega$ in (42) is much smaller in BCS-domain than in BEC-domain (for instance in superfluid ${ }^{3} \mathrm{He}-\mathrm{A}: \frac{\Delta_{0}}{\varepsilon_{F}} \sim \frac{T_{C}}{\varepsilon_{F}} \sim 10^{-3}$ and thus $\left.\frac{\left(\rho-C_{0}\right)}{\rho} \sim 10^{-6}\right)$.

The same estimate for the density of intrinsic angular momentum yields $\vec{L}=$ $\frac{\hbar}{2 m}\left(\rho-C_{0}\right) \vec{l}$. Note that even in BCS-case different calculations yield here different results. For $\vec{l}=$ const the evaluations of [51-57] yield $\vec{L}=\frac{\hbar}{2 m} \rho \vec{l}$ while the inclusion of the inhomogeneous textures of $\vec{l}$-vector restores $\vec{L}=\frac{\hbar}{2 m}\left(\rho-C_{0}\right) \vec{l}$.

Note that accordingly to Leggett [58] the total $N$-particles Hamiltonian $\hat{H}$ exactly commutes with the $z$-projection of the angular momentum $\hat{L}_{z}=\frac{\hbar}{2} \hat{N}$. This fact is in favor of the result $\vec{L}=\frac{\hbar}{2 m} \rho \vec{l}$ for $\vec{l}=$ const.

Returning back to the complicated problem of chiral anomaly let us recollect the two different approaches to this problem worked out in the end of eighties.

\section{Supersymmetric Hydrodynamics of the A1-Phase}

The first approach [7] is based on supersymmetric hydrodynamics of the A1-phase. 
Fig. 4 The qualitative illustration of fermionic $\left(S_{F}\right)$ and bosonic $\left(S_{B}\right)$ contributions to the total hydrodynamic action $S_{\text {tot }}$ of the A1-phase at $T \rightarrow 0$

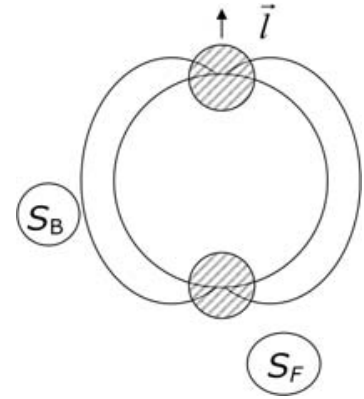

The idea of [7] was to check whether the chiral anomaly (or more precisely a term $\vec{j}_{a n} \vec{v}_{S}$ in total energy) is directly connected with the zeroes of the gap. The authors of [7] assumed that in 3D condensed matter system at low frequencies the only physical reason for anomaly can be connected with the infrared singularity. Note that ultraviolet singularities are absent in condensed matter systems in contrast with quantum electrodynamics. Strong (critical) fluctuations are also suppressed in 3D system. Thus the main idea of [7] was to check the dangerous infrared regions where the gap is practically zero. To do that the authors of [7] consider the total hydrodynamic action $S_{\text {tot }}$ of the A1-phase for low frequencies and small $q$-vectors as a sum of bosonic and fermionic contributions:

$$
S_{t o t}=S_{B}+S_{F}
$$

where $S_{B}\left(\rho, \vec{l}, \vec{v}_{S}\right)$ is a bosonic action and $S_{F}$ is a fermionic action connected with the zeroes of a superfluid gap (see Fig. 4).

Generally speaking the idea of [7] was to describe by the supersymmetric hydrodynamics all the zero energy Goldstone modes including the fermionic Goldstone mode which comes from the zero of the gap.

The authors of [7] were motivated by the nice paper [59] where for the first time a massless fermionic neutrino was included in the effective infrared Lagrangian for electro-weak interactions.

To be more specific bosonic action $S_{B}$ in (43) describes sound waves and orbital waves in purely bosonic limit and does not contain an anomalous term $\vec{j}_{a n} \vec{v}_{S}$ in the bosonic energy. Formally $S_{B}=\int L_{B} d^{4} x$, where $x=(\vec{r}, t)$ and bosonic Lagrangian $L_{B}=\vec{L} \dot{\vec{\theta}}-E_{B}$ where $\dot{\vec{\theta}}$-is angular frequency. It corresponds to bosonic energy $E_{B}$ which has the form:

$$
E_{B}=E_{O B}+\frac{j_{B}^{2}}{2 \rho},
$$

where $E_{O B}$ is connected with thermodynamic and liquid crystal like orbital energy. Thus an action $S_{B}$ describes 3 equations for bosonic hydrodynamics at $T=0$; namely the equation of mass-conservation:

$$
\partial \rho / \partial t+\operatorname{div} \vec{j}_{B}=0
$$


the equation for $\vec{l}$-vector dynamics:

$$
\dot{\vec{l}}+(\vec{v} S \vec{\nabla}) \vec{l}+\frac{2 m}{\hbar \rho}\left[\vec{l}, \frac{\partial E_{O B}}{\partial \vec{l}}-\partial_{i} \frac{\partial E_{O B}}{\partial_{i} \vec{l}}\right]=0,
$$

and the equation for superfluid velocity dynamics:

$$
\dot{\vec{v}}_{S}+\vec{\nabla}\left(\mu+\frac{v_{S}^{2}}{2}+\frac{\hbar}{4 m}\left(\vec{l} \operatorname{rot} \vec{v}_{S}\right)\right)=\frac{\hbar}{2 m} \vec{\nabla} l_{p}[\dot{\vec{l}}]_{p} .
$$

The right-hand side of the last equation expresses the famous Mermin-Ho identity for ${ }^{3} \mathrm{He}-\mathrm{A}$ [20]. The system of these 3 equations are in agreement with the equations of mass-current conservation $\frac{\partial j_{i}^{B}}{\partial t}+\frac{\partial}{\partial x} \Pi_{i n}=0$ and the equation of total energy conservation $\frac{\partial E_{B}}{\partial t}+\operatorname{div} \vec{Q}=0$. They contain sound wave $\omega=c_{S} k$ and orbital wave $\omega \sim k^{2} / m$.

Fermionic part of the action in (43) describes fermionic Goldstone mode and has the form:

$$
S_{F}=\int d^{4} x \Psi^{*} \hat{\Lambda} \Psi
$$

where doublets $\Psi^{*}$ and $\Psi$ are fermionic (grassman) variables and

$$
\begin{aligned}
\hat{\Lambda}=i \frac{\partial}{\partial t} & +\left(\begin{array}{cc}
i v_{l} \vec{l}\left(\vec{\nabla}-i p_{F} \vec{l}\right) & i v_{t} \vec{\Delta} \vec{\nabla} \\
i v_{t} \vec{\Delta} * \vec{\nabla} & -i v_{l} \vec{l}\left(\vec{\nabla}+i p_{F} \vec{l}\right)
\end{array}\right) \\
& +\left(\begin{array}{cc}
i \frac{v_{l}}{2} \operatorname{div} \vec{l} & i \frac{v_{t}}{2} \operatorname{div} \vec{\Delta} \\
i \frac{v_{t}}{2} \operatorname{div} \vec{\Delta}^{*} & -i \frac{v_{l}}{2} \operatorname{div} \vec{l}
\end{array}\right)+a(\vec{l} \operatorname{rot} \vec{l})+b\left(\vec{v}_{S} \vec{l}\right),
\end{aligned}
$$

where $\hat{\Lambda}$ is Lagrangian operator for nodal fermions. Here $v_{t}, v_{L}, a, b$-are phenomenological coefficients. This Lagrangian is valid both in weak and strong-coupling limits. In weak-coupling case $v_{t} \sim \Delta_{0} / \varepsilon_{F} v_{F}, v_{l} \sim v_{F}$-for "transverse" and "longitudial" Fermi-velocities, $\vec{\Delta}=\left(\vec{e}_{1}+i \vec{e}_{2}\right) \Delta_{0}$ and $\vec{v}_{S}=h / 2 m e_{1 i} \vec{\nabla} e_{2 i}$. The long derivatives $\left(\vec{\nabla} \pm i p_{F} \vec{l}\right)$ describe fermionic Goldstone variables with practically zero energy near north $\Psi_{1}\left(\vec{p} \approx p_{F} \vec{l}\right)$ and south $\Psi_{2}\left(\vec{p} \approx-p_{F} \vec{l}\right)$ poles. Remind that the quasiparticle energy: $E_{p}=\sqrt{\xi^{2}(p)+\frac{\Delta_{0}^{2}[\vec{l} \vec{p}]^{2}}{p_{F}^{2}}}=0$ for $\vec{p}= \pm p_{F} \vec{l}$. Accordingly the doublet for $\Psi$ has the form $\Psi=\left(\begin{array}{l}\Psi_{1} \\ \Psi_{2}^{*}\end{array}\right)$.

To come from our supersymmetric action in (43) to a standard action which contains only bosonic fields we must eliminate fermionic degrees of freedom in (39) by calculating the functional integral over Grassman variables. This yields:

$$
S_{e f f}=S_{B}+\Delta S_{B}
$$

where $\Delta S_{B}=\ln \int D \Psi D \Psi^{*} \exp \left(\int d^{4} x \Psi^{*} \hat{\Lambda} \Psi\right)=\ln \operatorname{Det}\left(\hat{\Lambda} \hat{\Lambda}_{0}^{-1}\right)=\operatorname{Tr} \ln \left(\hat{\Lambda} \hat{\Lambda}_{0}^{-1}\right)$, and $\hat{\Lambda}_{0}$ is normalizing operator.

In hydrodynamic ideology it coincides with $\hat{\Lambda}$ in homogeneous case, where $\vec{l}=$ const and $\vec{\Delta}=\Delta_{0}\left(\vec{e}_{1}+i \vec{e}_{2}\right)=$ const. 
If we hope to obtain an anomalous term in current $\vec{j}_{\text {an }}=-\frac{\hbar}{4 m} C_{0}(\vec{l} \operatorname{rot} \vec{l}) \vec{l}$ with large coefficient $C_{0} \sim \rho$ we must get the term $\vec{j}_{a n} \vec{v}_{S}$ in $\Delta L_{B}\left(\Delta S_{B}=\int \Delta L_{B} d^{4} x\right)$. But due to small fermionic density $\rho_{F} \sim \Psi^{*} \Psi$ near the south and north poles (small statistical weight of fermionic pockets on Fig. 4 in comparison with total density $\rho$ ) we have to get $C_{0} \sim \delta\left(\vec{p} \pm p_{F} \vec{l}\right)$ in momentum space or accordingly to $C_{0}=$ const in real space. That is why in order to obtain an anomalous term in current we must find very strong delta-functional infra-red divergencies in $\Delta S_{B}$.

But a very accurate calculation of the functional integral (of $\operatorname{Tr} \ln \hat{\Lambda} \hat{\Lambda}_{0}^{-1}$ ) in hydrodynamic limit $(\omega \rightarrow 0, k \rightarrow 0$ or accordingly $\vec{r}$ and $t \rightarrow \infty)$ will give us a final result:

$$
\Delta L_{B}=\frac{p_{F}^{2} v_{L}}{32 \pi^{2}}\left\{[\vec{l} \operatorname{rot} \vec{l}]^{2}+\frac{v_{t}^{2}}{v_{l}^{2}}(\vec{l} \operatorname{rot} \vec{l})^{2}\right\} \ln \frac{x_{\max }}{x},
$$

where $x_{\text {max }} \sim l_{M F}$ in hydrodynamic regime at $T=0, l_{M F}$-is a mean-free path, $\xi_{0}<x<l_{M F}, \xi_{0} \sim \frac{v_{F}}{\Delta_{0}}$ is a coherence length.

It means that only weak logarithmic type of infra-red singularities is present in the system and we do not have in it a strong $\delta$-functional singularity.

These weakly (logarithmically) divergent terms lead only to a strong renormalization of liquid crystal type coefficients $K_{2}$ and $K_{3}$ in $E_{O B}[60,61]$ (and after Fourier transform in the spectrum of orbital waves $\omega \sim \frac{q^{2}}{m} \ln \frac{\Delta_{0}}{v_{F} q}$ ) but they do not lead to the appearance of large anomalous coefficient $C_{0}$ and thus do not destroy the hydrodynamics. Hence even if chiral anomaly exists in the BCS-domain of 3D A1-phase, it is not directly connected with the dangerous regions of momentum space near zeroes of the gap (it does not have an infra-red character).

\section{The Different Approach Based on the Formal Analogy with Quantum Electrodynamics}

The authors of [8-12] have the different also rather nice approach based on the formal analogy between the anomalous current in 3D ${ }^{3} \mathrm{He}-\mathrm{A}$ and chiral anomaly in QEDtheory. They assume that anomalous current with the coefficient $C_{0} \sim \rho$ in BCSdomain of the A1-phase is not directly connected with the zeroes of the gap (thus it is not contained even in the supersymmetric hydrodynamics). They think that it is connected with the global topological considerations and thus should be added to (43) by hands. To illustrate this point they solve microscopic Bogolubov-de-Gennes (BdG) equations for fermionic quasiparticles in a given twisted texture $(\vec{l} \| \operatorname{rot} \vec{l})$ of the $\vec{l}$-vector. To be more specific they consider the case:

$$
\vec{l}=\vec{l}_{0}+\partial \vec{l}
$$

where

$$
l_{z}=l_{0 z}=e_{z} ; \quad l_{y}=\delta l_{y}=B x ; \quad l_{x}=0 .
$$

In this case:

$$
\vec{l} \operatorname{rot} \vec{l}=l_{z} \frac{\partial l_{y}}{\partial x}=B=\mathrm{const}
$$


and accordingly

$$
\vec{j}_{a n}=-\frac{\hbar}{4 m} C_{0} B \vec{e}_{z} .
$$

After linearization BdG equations become equivalent to Dirac equation in homogeneous magnetic field $B=(\vec{l} \operatorname{rot} \vec{l})$.

Namely after linearization $\mathrm{BdG}$ equation reads $\hat{H} \chi=E \chi$, where the doublet $\chi$ has the form $\chi=\left(\begin{array}{c}u(x) \\ v(x)\end{array}\right) \exp \left(i p_{z} z+i p_{y} y\right)$ and for the Hamiltonian we have:

$$
\hat{H}=\xi\left(p_{z}\right) \hat{\sigma}_{3}+v_{\perp}\left(\hat{\sigma}_{1} \frac{1}{i} \partial_{x}-\hat{\sigma}_{2}\left(p_{y}-e B x\right)\right),
$$

where $\left\{\hat{\sigma}_{3}, \hat{\sigma}_{2}, \hat{\sigma}_{1}\right\}$-are Pauli matrices, $\xi\left(p_{z}\right)=\frac{p_{z}^{2}-p_{F}^{2}}{2 m}, v_{t}=v_{F} \frac{\Delta_{0}}{\varepsilon_{F}}$ —corresponds to weak-coupling limit, and $e=\frac{p_{Z}}{p_{F}}= \pm 1$-is an electric charge.

The solution of $\mathrm{BdG}$ equations yields for the doublets $\chi(x)$ :

$$
\chi_{n_{L}}(x)=\theta(-e B)\left(\begin{array}{c}
\alpha_{n_{L}} f_{n_{L}}(\tilde{x}) \\
i \beta_{n_{L}} f_{n_{L}-1}(\tilde{x})
\end{array}\right)+\theta(e B)\left(\begin{array}{c}
\alpha_{n_{L}} f_{n_{L}-1}(\tilde{x}) \\
i \beta_{n_{L}} f_{n_{L}}(\tilde{x})
\end{array}\right),
$$

where $\theta$-is a step-function, $f_{n_{L}}(\tilde{x})=f_{n_{L}}\left(x-\frac{p_{y}}{e B}\right)$-is the ortho-normalized wavefunction of harmonic oscillator, $f_{-1}=0,\left|\alpha_{n_{L}}\right|^{2}=\frac{E_{n_{L}}+\xi\left(p_{z}\right)}{2 E_{n_{L}}},\left|\beta_{n_{L}}\right|^{2}=\frac{E_{n_{L}}-\xi\left(p_{z}\right)}{2 E_{n_{L}}}-$ are Bogolubov coefficients $\left(\left|\alpha_{n_{L}}\right|^{2}+\left|\beta_{n_{L}}\right|^{2}=1\right)$.

Accordingly for the spectrum:

$$
E_{n_{L}}\left(p_{z}\right)= \pm \sqrt{\xi^{2}\left(p_{z}\right)+\tilde{\Delta}_{n_{L}}^{2}}
$$

where $\tilde{\Delta}_{n_{L}}^{2}=2 n_{L} v_{t}^{2} p_{F}|e B|$ is a gap squared and $n_{L}$ is a quantum number for Landau level. The solution for $\chi_{n_{L}}$ and $E_{n_{L}}$ contains the level asymmetry.

Namely for $n_{L} \neq 0$ (see Fig. 5) all the levels are gapped $\tilde{\Delta}_{n_{L}} \neq 0$ and doubly degenerate with respect to $p_{z} \rightarrow-p_{z}$. Their contribution to total mass-current is zero for $T \rightarrow 0$.

However for $n_{L}=0$ there is no gap $\tilde{\Delta}_{0}=0$ and we have an asymmetric chiral branch which exists only for $p_{z}<0$ (only for one sign of $e B$ ). The energy spectrum for $n_{L}=0$ yields:

$$
E_{0}=\xi\left(p_{z}\right)
$$

We can say that there is no gap for zeroth Landau level. Moreover in BCS-domain $E_{0}=0$ for $\left|p_{z}\right|=p_{F}$-the chiral level crosses the origin in Fig. 5, so we have a zero mode.

Note that in BEC-domain $E_{0} \geq|\mu|$ and the zeroth Landau level does not cross the origin. The absence of a zero-mode in BEC-domain is a physical reason why $C_{0}=0$ here.

The zeroth Landau level gives an anomalous contribution to the total current in BCS-domain:

$$
\vec{j}_{a n}(\vec{r}=0)=-\vec{e}_{z}(\vec{l} \operatorname{rot} \vec{l}) \int_{p_{z}<0} \frac{p_{z}}{2 \pi^{2}} d \xi\left(p_{z}\right)=-\frac{\hbar C_{0}}{4 m}(\vec{l} \operatorname{rot} \vec{l}) \vec{l},
$$


Fig. 5 (Color online) The level structure of the Dirac equation in magnetic field $B=(\vec{l} \operatorname{rot} \vec{l})$. All the levels with $n_{L} \neq 0$ are doubly degenerate. The zeroth level is chiral. It crosses the origin for $\left|p_{z}\right|=p_{F}$ in BCS-domain $(\mu>0)$. We also illustrate the concept of the spectral flow which will be discussed in Sect. 9

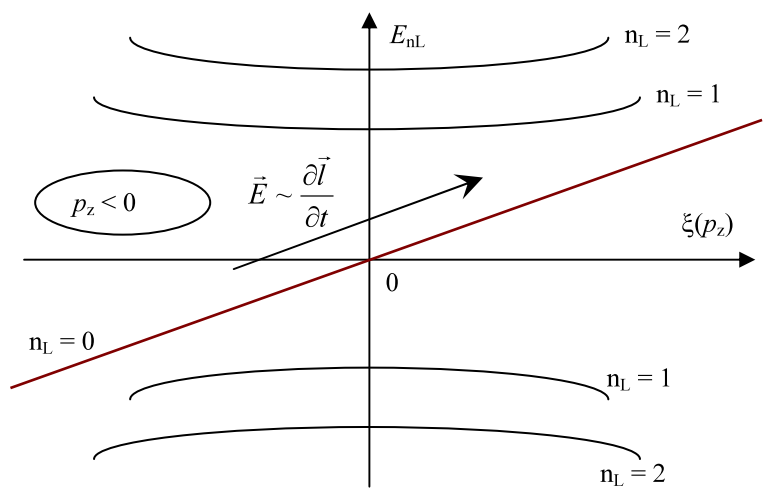

where

$$
\frac{(\vec{l} \operatorname{rot} \vec{l}) p_{z}}{2 \pi^{2} p_{F}}=\frac{e B}{2 \pi^{2}}=\int\left|f_{0}\right|^{2} \frac{d p_{y}}{2 \pi}
$$

and as a result:

$$
C_{0} \approx m \frac{p_{F}^{3}}{6 \pi^{2}} \approx \rho
$$

in BCS-domain.

It is easy to observe that the integral for $C_{0}$ in (60), (61) is governed by the narrow cylindrical tube inside the Fermi-sphere (see Fig. 6) with the length $p_{F}$ parallel to the $\vec{l}$-vector and radius of the cylinder squared given by:

$$
\left\langle p_{y}^{2}\right\rangle \sim p_{F}|e B| .
$$

According to the ideas of [8-10, 37-40] this tube plays the role of a vortex in momentum space, thus providing a normal core and anomalous current at $T=0$.

Note that a key result of [8-12] connected with the absence of the gap for the energy of zeroth Landau level (see (59)) in pretty stable with respect to small modifications of the texture of the $\vec{l}$-vector in (53). The careful analysis fulfilled by the authors of present paper shows that an account of small bending corrections with $[\vec{l} \operatorname{rot} \vec{l}] \neq 0$ (small tilting of magnetic field with respect to $(x, y)$ plane $\left.\vec{B}=B_{0} \vec{e}_{z}+B_{1} \vec{e}_{x}\right)$ as well as small inhomogeneties of a magnetic field $B=B_{0}+B_{1} x$, which lead to the double-well effective potential, do not suppress a zero-mode in the spectrum of BdGequation (do not lead to the appearance of a gap $\tilde{\Delta}_{n=0}$ for zeroth Landau level).

\section{How to Reach Hydrodynamic Regime $\omega \tau \ll 1$}

Inspite of the zero-mode stability the authors of [7] expressed their doubts with respect to the calculation of $C_{0}$ based on Dirac equation in homogeneous magnetic field $B=(\vec{l} \operatorname{rot} \vec{l})$. From their point of view the calculation of $C_{0}$ from (60), (61) is an oversimplification of a difficult many-particle problem. In particular they emphasized the role of the finite damping $\gamma=1 / \tau$ as well as other residual interactions 
Fig. 6 The contribution to the coefficient $C_{0}$ is governed by the narrow cylindrical tube of the length $p_{F}$ and the width $\left\langle p_{y}^{2}\right\rangle \sim p_{F}|e B|$ inside the Fermi-sphere
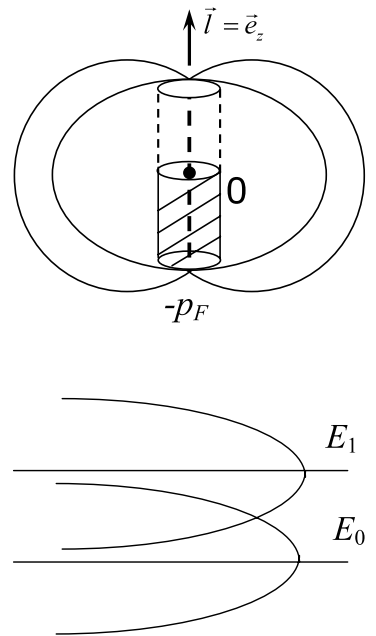

Fig. 7 The possible role of the damping in reaching hydrodynamic limit for low frequencies and small $\vec{k}$-vectors when $\gamma>\omega_{0}$ $\left(\omega_{0}=E_{1}-E_{0}\right.$-is level spacing)

Fig. 8 Different decay processes for damping of chiral fermions at $T=0$ : the standard three-fermion decay process and a decay process with the emission of orbital wave
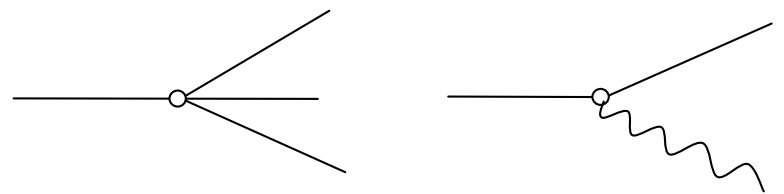

to destroy the chiral anomaly, which is connected with the states inside the Fermi sphere, thus restoring the superfluid hydrodynamics (without normal velocity $\vec{v}_{n}$ and normal density $\rho_{n}$ ). Indeed, if the damping $\gamma$ is larger than the level spacing of the Dirac equation:

$$
\omega_{0}=v_{t} p_{F} \sqrt{\frac{|\vec{l} \operatorname{rot} \vec{l}|}{p_{F}}}
$$

in case when $\xi\left(p_{z}\right)=0$, then the contribution from the zeroth Landau level should be washed out by damping (see Fig. 7) and we will reach hydrodynamic regime. The damping $\gamma$ for chiral fermions (for fermions living close to the nodes) in a very clean A1-phase without impurities is defined at $T=0$ by the different decay processes (see [48]).

It is natural to assume that the only parameter which defines $\gamma$ at $T=0$ for chiral fermions is $\Delta_{0}\langle\theta\rangle=\Delta_{0} \frac{\left\langle p_{\perp}\right\rangle}{p_{F}}$. The leading term in decay processes is given by the emission of an orbital wave (see Fig. 8). It reads

$$
\gamma \propto\left[\frac{\Delta_{0}^{2} p_{\perp}^{2} / p_{F}^{2}+v_{F}^{2}\left(p_{z}-p_{F}\right)^{2}}{\varepsilon_{F}}\right] .
$$

For $p_{z}=p_{F}\left(\xi\left(p_{z}\right)=0\right)$ :

$$
\gamma \sim \frac{\Delta_{0}^{2}}{\varepsilon_{F}} \frac{p_{\perp}^{2}}{p_{F}^{2}}
$$


Note that for chiral fermions on zeroth Landau level

$$
\frac{\left\langle p_{\perp}\right\rangle}{p_{F}}=\left(\frac{|\vec{l} \operatorname{rot} \vec{l}|}{p_{F}}\right)^{1 / 2}
$$

and the level spacing for $\xi\left(p_{z}\right)=0$ reads:

$$
\omega_{0} \sim \Delta_{0} \frac{\left\langle p_{\perp}\right\rangle}{p_{F}} .
$$

Hence $\gamma / \omega_{0} \ll 1$ close to zero mode for these two decay processes and we are in a ballistic regime. Thus it is difficult to wash out the contribution form the zeroth Landau level by the different decay processes in superclean ${ }^{3} \mathrm{He}-\mathrm{A} 1$-phase at $T=0$. Note that a hydrodynamic regime $\omega \tau \ll 1$ could be easily reached in the presence of non-magnetic impurities or in the presence of aerogel [62-65].

\subsection{The Concept of Spectral Flow and Exact Anomalies Cancellation}

If anomalous current exists in superclean A1-phase at $T=0$ it should be compensated somehow. According to [8-10] the deficit in the equation for the conservation of total linear momentum due to the presence of anomalous current:

$$
\frac{\partial j_{a n}^{i}}{\partial t}+\frac{\partial \Pi_{i k}}{\partial x_{k}}=I,
$$

where $I=\frac{3 \hbar}{4 m} C_{0} \vec{l}\left(\operatorname{rot} \vec{l} \frac{\partial \vec{l}}{\partial t}\right)$, is exactly compensated by quasiparticle contribution $\vec{P}_{\text {quas }}$ :

$$
\frac{\partial \mathrm{P}_{q u a s}^{i}}{\partial t}+\frac{\partial \Phi_{i k}}{\partial x_{k}}=-I,
$$

where $\vec{P}_{\text {quas }}=\rho_{n}(T=0)\left(\vec{v}_{n}-\vec{v}_{S}\right)$ in hydrodynamic regime.

Note that $\rho_{n}(T=0) \sim \frac{|[\vec{l} \operatorname{rot} \vec{l}]|}{\Delta_{0}}$ is non-analytical and connected with non-zero bending. The arguments of [8-10] are connected with the non-conservation of axial current $j_{5}$ in QED which just compensates $I$ via a Schwinger term $\vec{E} \vec{B} \sim \frac{\partial \vec{l}}{\partial t} \operatorname{rot} \vec{l}$. Physically, according to [8-10, 66, 67], this cancellation is due to the spectral flow from negative to positive energy values along the anomalous branch with $n_{L}=0$ on Fig. 5 and then to the quasiparticle bath in the presence of an electric field $\vec{E} \sim \frac{\partial \vec{l}}{\partial t}$ (of a time-dependent texture of the $\vec{l}$-vector).

Note that in the physics of a vortex-core in case of cylindrical symmetry there is one anomalous level which crosses the zero energy (see Fig. 9). As a function of generalized angular momentum $Q$ it represents at $T=0$ the set of discrete points separated by a minigap $\omega_{0} \sim \Delta_{0}^{2} / \varepsilon_{F}$. Thus at $T=0$ and in a superclean case $\gamma=$ $1 / \tau \rightarrow 0$ the spectral flow from negative to positive energies is totally suppressed. Thus the Thouless result [68] for the Berry-phase without anomaly is restored for the physics of vortex friction. An inclusion of large number of impurities or finite temperature leads to the revival of anomaly in hydrodynamic regime $\omega_{0} \tau \ll 1$ in 
Fig. 9 (Color online) Levels structure in the vortex core of

${ }^{3} \mathrm{He}-\mathrm{A}$. All the branches are even in generalized angular momentum $Q$, but one branch $E\left(p_{z}=0, Q\right)=-\omega_{0} Q$ which crosses zero energy at $Q=0$ is chiral (odd in $Q$ ). It participates in momentum exchange between fermions in the vortex core and fermions of the heat bath in the hydrodynamic limit $\omega_{0} \tau \ll 1$ accordingly to [37-40]

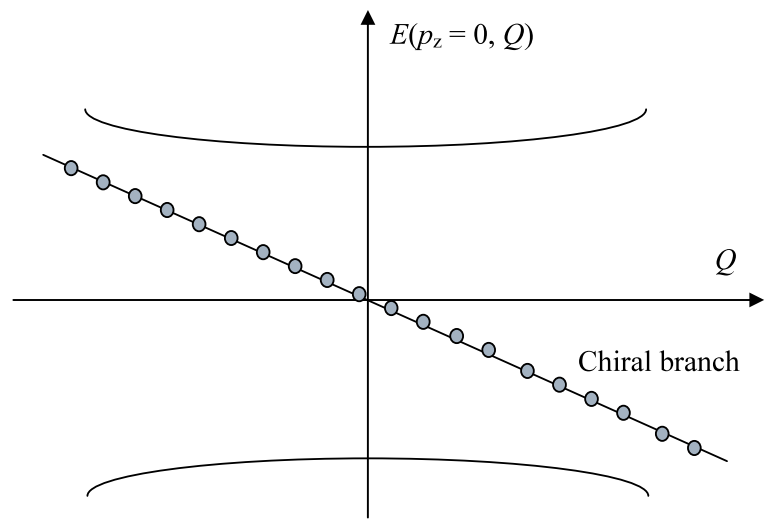

case of vortices. So, we could assume that the chiral anomaly and the spectral flow are ineffective at $T=0$ both for vortices and for a bulk A-phase of superfluid ${ }^{3} \mathrm{He}$ in a superclean limit. Thus the question how the total linear momentum is conserved in this case in an infinite system (without the walls) is very untrivial and unresolved yet.

We think that exact cancellation between time-derivatives of anomalous and quasiparticle currents should be demonstrated explicitly by the derivation and solution of kinetic equations for nodal quasiparticles both in ballistic and hydrodynamic regimes. Note that if $T \neq 0$ (as we always have in real experiment) and for low frequencies $\omega \tau(T \neq 0) \ll 1\left(\tau(T \neq 0) \sim \alpha T^{-n}\right)$ a relative normal velocity $\vec{v}_{n}-\vec{v}_{S}=\frac{\partial \varepsilon_{0}}{\partial \overrightarrow{\mathrm{P}}_{\text {quas }}}$ becomes an additional hydrodynamic variable and so the cancellation of the linear momentum deficit will be fulfilled automatically.

Thus the question with exact anomalies compensation exists only for $T=0$. Note that an approach based on kinetic equation for quasiparticles at different temperatures and impurities concentrations in a vortex core of $s$-wave superconductors and superfluid ${ }^{3} \mathrm{He}$ was worked out by [69-72] for the case of a singular vortex.

In case of nonsingular vortex structures in ${ }^{3} \mathrm{He}-\mathrm{A}$ we should mention here also the articles of $[66,67]$ where in finite systems the author consider the scattering of quasiparticles on the walls of the container to get a finite $\gamma$ at $T=0$. The importance of prehistory of orbital texture in the framework of the concept of a spectral flow was also stressed in these papers.

\section{Conclusions}

We solve the Leggett equations and construct the phase-diagram of BCS-BEC crossover at low temperatures $T \ll T_{C}$ for $100 \%$-polarized 3D A1-phase. From the evaluation of low temperature specific heat and normal density we see the indications of quantum phase - transition close to the point $\mu(T=0)=0$. In the same time deep in BCS and BEC-domains the crossover ideas of Leggett and Nozieres, Schmitt-Rink work pretty well. In these regions the phase-diagram for $p$-wave gas resembles in gross features the $s$-wave case. We discuss a complicated problem of chiral anomaly and mass-current non-conservation in BCS A1-phase at $T=0$. We present two 
different approaches to this problem-one based on supersymmetric hydrodynamics, another one-on the formal analogy with Dirac equation in QED-theory. We evaluate the damping $\gamma=1 / \tau$ due to different decay processes in superclean BCS A1-phase at $T=0$ and find that $\gamma$ is small in comparison with level spacing $\omega_{0}$ of BdG-equation. To reach the hydrodynamic regime $\omega \tau \ll 1$ we need a sufficient amount of aerogel or nonmagnetic impurities at $T=0$. We assume that both in hydrodynamic and in a ballistic regimes at $T=0$ we have to derive a reliable kinetic equation to demonstrate explicitly an exact cancellation between time-derivatives of anomalous current $\vec{j}_{a n}=-\frac{\hbar}{4 m} C_{0} \vec{l}(\vec{l} \operatorname{rot} \vec{l})$ and quasiparticle contribution $\vec{P}_{\text {quas }}$ in the equation for the conservation of the total linear momentum $\vec{j}_{\text {tot }}$. Note that for the full theoretical analysis of the problem the other residual interactions different from damping are also important for nodal fermions. To check whether a chiral anomaly has an infra-red manifestation (which was not caught in the approach based on supersymmetric hydrodynamics of [7]) it will be useful to derive a complete set of Ward identities between self-energies of chiral fermions $\Sigma$ and the corresponding vertices $\Gamma$. The idea is to find in this approach either a strong infra-red singularity or a powerful reexpansion of quasiparticle spectrum for $\omega, \vec{k} \rightarrow 0$.

Note that the importance of residual Fermi-liquid like interactions for the analysis of half-integer vortex in 3D A-phase of ${ }^{3} \mathrm{He}$ was recently emphasized in [73].

We invite experimentalists to enter this very interesting problem. It will be interesting to measure a spectrum and damping of orbital waves in superfluid A-phase of ${ }^{3} \mathrm{He}$ at low temperatures $T \ll T_{C}$. The spectrum is quadratic for low frequencies $\omega<\Delta_{0}^{2} / \varepsilon_{F}$ and contains a density of intrinsic angular momentum as a coefficient near the linear in frequency term:

$$
\frac{\left(\rho-C_{0}\right)}{\rho} \omega \sim \frac{k_{Z}^{2}}{m} \ln \frac{\Delta_{0}}{v_{F} k_{Z}},
$$

where $\vec{e}_{z} \| \vec{l}$.

At higher frequencies the spectrum is almost linear $\omega^{2} \ln \frac{\Delta_{0}}{\omega} \sim v_{F}^{2} k_{Z}^{2} \ln \frac{\Delta_{0}}{v_{F} k_{Z}}$ $[49,50]$.

The damping of the orbital waves provides an evaluation of orbital viscosity in ${ }^{3} \mathrm{He}-\mathrm{A}$ at low temperatures $T \ll T_{C}$. Note that even in this case it is an interacting possibility to get an overdamped (diffusive) character of the spectrum.

This possibility gains a theoretical support in papers of [74-76] where in the functional integral technique in the hydrodynamic limit of small $\omega$ and $\vec{k}$ the author obtained several overdamped modes in partially polarized A1-phase.

Another possibility of an overdamped diffusive spectrum was considered by $[77,78]$ in the impurity diagrammatic technique [79, 80] for a hydrodynamic regime $\omega \tau \ll 1$ of spin waves in a frustrated 2D AFM. Note that in the opposite high-frequency regime the spectrum of spin waves is linear. Here it is possible to extend experiments of orbital inertia and orbital viscosity for nonsingular vortices in A-phase of ${ }^{3} \mathrm{He}$ on low temperatures $T \ll T_{C}$. Of course, to have A-phase at low temperatures we need a strong spin-polarization.

Note also that a crossover from the ballistic to the hydrodynamic regime $\omega \tau \ll 1$ could be fulfilled both due to aerogel (nonmagnetic impurities) or due to the final 
temperature $T \neq 0$ which is always present in a real experiment. In the last case the damping $\gamma \sim T^{n}$ is temperature dependent.

Finally, to measure non-trivial topological effects in 2D we propose to perform the experiments with a Josephson current between two thin films or two magnetic taps: one with a 2D axial phase and topological charge $Q=1$ [81] and another one with planar 2D-phase with $Q=0$. In this type of experiments we hope it will be possible to measure directly $\Delta Q=1$.

Another possibility is to measure the contribution of massless Majorana fermions for the edge states on the surface of superfluid ${ }^{3} \mathrm{He}-\mathrm{B}$ and the rough wall (or on the surface of a vibrating wire in Lancaster experiments) [82, 83].

Acknowledgements The authors acknowledge interesting and useful discussion with A.S. Alexandrov, A.F. Andreev, P.N. Brusov, Yu.M. Bunkov, A.V. Chubukov, V.V. Dmitriev, A. Golov, V. Gurarie, I.A. Fomin, W. Halperin, Yu. Kagan, W. Ketterle, K.Yu. Kovalev, D.M. Lee, B.E. Meierovich, V.M. Osadchiev, L.P. Pitaevskii, G.V. Shlyapnikov, M. Stone, V.V. Val'kov, G.E. Volovik, and are grateful to RFBR (grant \# 08-02-00224) for financial support of this work.

Open Access This article is distributed under the terms of the Creative Commons Attribution Noncommercial License which permits any noncommercial use, distribution, and reproduction in any medium, provided the original author(s) and source are credited.

\section{References}

1. C. Ticknor, C.A. Regal, D.S. Jin, J.L. Bohn, Phys. Rev. A 69, 042712 (2004)

2. C.A. Regal, C. Ticknor, J.L. Bohn, D.S. Jin, Phys. Rev. Lett. 90, 053201 (2003)

3. C.H. Schunck, M.W. Zwierlein, C.A. Stan, S.M.F. Raupach, W. Ketterle, A. Simoni, E. Tiesinga, C.J. Williams, P.S. Julienne, Phys. Rev. A 71, 045601 (2005)

4. Y. Inada, M. Horikoshi, S. Nakajima, M. Kuwata-Gonokami, M. Ueda, T. Mukaiyama, arXiv:0803.1405 [cond-mat] (2008)

5. J. Fuchs, C. Ticknor, P. Dyke, G. Veeravalli, E. Kuhnle, W. Rowlands, P. Hannaford, C.J. Vale, arXiv:0802.3262 [cond-mat] (2008)

6. J.P. Gaebler, J.T. Stewart, J.L. Bohn, D.S. Jin, Phys. Rev. Lett. 98, 200403 (2007)

7. A.F. Andreev, M.Yu. Kagan, Sov. Phys. JETP 66, 594 (1987)

8. A.V. Balatskii, G.E. Volovik, V.A. Konyshev, Sov. Phys. JETP 63, 1194 (1986)

9. G.E. Volovik, JETP Lett. 43, 428 (1986)

10. G.E. Volovik, V.P. Mineev, Sov. Phys. JETP 83, 1025 (1982)

11. R. Combescot, T. Dombre, Phys. Rev. B 33, 79 (1986)

12. R. Combescot, T. Dombre, Phys. Rev. B 28, 5140 (1983)

13. H.-Y. Kee, A. Raghavan, K. Maki, arXiv:0711.0929 [cond-mat] (2007)

14. R. Roy, C. Kallin, Phys. Rev. B 77, 174513 (2008)

15. D.A. Ivanov, Phys. Rev. Lett. 89, 208 (2001)

16. K.S. Novoselov, A.K. Geim, S.V. Morozov, D. Jiang, M.I. Katsnelson, I.V. Grigorieva, S.V. Dubonos, A.A. Firsov, Nature 438, 197 (2005)

17. N. Tajima, et al. Eur. Phys. Lett. 80, 47002 (2007)

18. T.D.C. Bevan, A.J. Manninen, J.B. Cook, H. Alles, J.R. Hook, H.E. Hall, J. Low Temp. Phys. 109, 423 (1997)

19. T.D.C. Bevan, A.J. Manninen, J.B. Cook, J.R. Hook, H.E. Hall, T. Vachaspati, G.E. Volovik, Nature 386, 689 (1997)

20. D. Vollhardt, P. Woelfle, The Superfluid Phases of Helium 3 (Taylor, London, 1990)

21. P.B. Littlewood, G. Varelogiannes (eds.), Anomalous Complex Superconductors, Proc. of the First Euroconference in Crete (North Holland, Amsterdam, 1999)

22. L.D. Landau, E.M. Lifshitz, Quantum mechanics, 3rd edn., vol. 3 (Pergamon, Oxford, 1991)

23. V. Gurarie, L. Radzihovsky, Ann. Phys. 322, 2 (2007) 
24. Chi-Ho Cheng, S.-K. Yip, Phys. Rev. Lett. 95, 070404 (2005)

25. F.R. Klinkhamer, G.E. Volovik, JETP Lett. 80, 389 (2004)

26. R. Combescot, X. Leyronas, M.Yu. Kagan, Phys. Rev. A 73, 023618 (2006)

27. I.V. Brodsky, M.Yu. Kagan, A.V. Klaptsov, R. Combescot, X. Leyronas, JETP Lett. 82, 306 (2005)

28. I.V. Brodsky, M.Yu. Kagan, A.V. Klaptsov, R. Combescot, X. Leyronas, Phys. Rev. A 73, 032724 (2006)

29. R. Combescot, M.Yu. Kagan, S. Stringary, Phys. Rev. A 74, 042717 (2006)

30. J.A. Hertz, Phys. Rev. B 14, 1165 (1976)

31. A.J. Millis, Phys. Rev. B 48, 7183 (1993)

32. L.P. Gor'kov, T.K. Melik-Barchudarov, Sov. Phys. JETP 13, 1018 (1961)

33. M.Yu. Kagan, A.V. Chubukov, JETP Lett. 47, 525 (1988) - this calculation was done for nonpolarized A-phase in the case when $s$-wave scattering is totally suppressed. The calculation for $100 \%$-polarized A1-phase yields only $10 \%$ difference from Kagan, Chubukov result for preexponential factor $0.1 \varepsilon_{F}$

34. M.A. Baranov, M.Yu. Kagan, Yu. Kagan, JETP Lett. (1996)

35. M.A. Baranov, M.Yu. Kagan, A.V. Chubukov, Int. J. Mod. Phys. B 6, 2471 (1992)

36. M.A. Baranov, D.V. Efremov, M.S. Mar'enko, M.Yu. Kagan, Sov. Phys. JETP 90, 861 (2000)

37. G.E. Volovik, The Universe in Helium Droplet (Oxford University Press, Oxford, 2002)

38. G.E. Volovik, Exotic Properties of Superfluid ${ }^{3} \mathrm{He}$ (World Scientific, Singapore, 1992)

39. G.E. Volovik, Quantum phase transitions from topology in momentum space, in Quantum Analogues: From Phase Transitions to Black Holes and Cosmology, ed. by W.G. Unruh, R. Schuetzhold. Springer Lecture Notes in Physics, vol. 718 (Springer, Berlin, 2007), pp. 31-73

40. G.E. Volovik, arXiv:cond-mat/0601372

41. N. Read, D. Green, Phys. Rev. B 61, 10267 (2000)

42. A.J. Leggett, Modern Trends in the Theory of Condensed Matter Physics (Springer, Berlin, 1980), pp. 13-22

43. M. Iskin, C.A.R. Sa de Melo, Phys. Rev. Lett. 96, 040402 (2006)

44. S.S. Botelho, C.A.R. Sa de Melo, J. Low Temp. Phys. 140, 409 (2005)

45. M.Yu. Kagan, S.L. Ogarkov, Laser Phys. 18, 509 (2008)

46. M.Yu. Kagan, D.V. Efremov, Sov. Phys. Low Temp. 85, 779 (2009)

47. M.Yu. Kagan, S.L. Ogarkov, J. Phys., Conf. Ser. 150, 032037 (2009)

48. E.M. Lifshitz, L.P. Pitaevskii, Statistical Physics, Part 2 (Nauka, Moscow, 1973)

49. V.G. Vaks, V.M. Galitskii, A.I. Larkin, Sov. Phys. JETP 42, 1319 (1962)

50. A.V. Chubukov, M.Yu. Kagan, unpublished

51. M. Stone, I. Anduaga, Ann. Phys. 323, 2 (2008)

52. M. Stone, F. Gaitan, Ann. Phys. 178, 89 (1987)

53. M. Stone, Phys. Rev. B 54, 13222 (1996)

54. M. Stone, Physica B 280, 117 (2000)

55. M. Stone, R. Roy, Phys. Rev. B 69, 184511 (2004)

56. F. Gaitan, Phys. Lett. A 151, 551 (1990)

57. N.D. Mermin, P. Muzikar, Phys. Rev. B 21, 980 (1980)

58. A.J. Leggett, private communication

59. D.V. Volkov, V.P. Akulov, JETP Lett. 16, 438 (1972)

60. M.C. Cross, J. Low Temp. Phys. 21, 525 (1975)

61. M.C. Cross, J. Low Temp. Phys. 26, 165 (1977)

62. V.V. Dmitriev, D.A. Krasnikhin, N. Mulders, V.V. Zavjalov, D.E. Zmeev, JETP Lett. 86, 594 (2007)

63. J. Elbs, Yu.M. Bunkov, E. Collin, H. Godfrin, G.E. Volovik, Phys. Rev. Lett. 100, 215304 (2008)

64. I.A. Fomin, JETP Lett. 88, 59 (2008)

65. I.A. Fomin, JETP Lett. 77, 240 (2003)

66. G.E. Volovik, Sov. Phys. JETP 102, 1838 (1992)

67. G.E. Volovik, JETP Lett. 61, 935 (1995)

68. D.J. Thouless, P. Ao, Q. Niu, Phys. Rev. Lett. 76, 3758 (1996)

69. N.B. Kopnin, M.M. Salomaa, Phys. Rev. B 44, 9667 (1991)

70. N.B. Kopnin, Physica B 210, 267 (1995)

71. N.B. Kopnin, Physica B 280, 231 (2000)

72. N.B. Kopnin, P.S. Soininen, M.M. Salomaa, Phys. Rev. B 45, 5491 (1992)

73. V. Vakaryak, A.J. Leggett, arXiv:0906.2631 [condmat]

74. P.N. Brusov, M.V. Lomakov, N.P. Brusova, Sov. Low Temp. Phys. 21, 85 (1995)

75. P.N. Brusov, M.V. Lomakov, J. Low Temp. Phys. 85, 91 (1991) 
76. P.N. Brusov, P.P. Brusov, Collective Excitations in the Unconventional Superconductors and Superfuids (World Scientific, New Jersey, 2009, in press)

77. A.V. Chubukov, Phys. Rev. B 44, 12318 (1991)

78. A.V. Chubukov, Phys. Rev. B 52, R3840 (1995)

79. A.A. Abrikosov, L.P. Gorkov, Sov. Phys. JETP 12, 1243 (1961)

80. A.I. Larkin, Sov. Phys. JETP 31, 784 (1970)

81. G.E. Volovik, A. Solov'ev, V.M. Yakovenko, JETP Lett. 49, 65 (1989)

82. G.E. Volovik, arXiv:0907.5389 [condmat] (2009)

83. G.E. Volovik, arXiv:0909.3084 [condmat] (2009) and references therein 